\title{
Discovery of Rodrigama Gauld in the Old World, with description of two new species (Hymenoptera, Ichneumonidae, Poemeniinae)
}

\author{
Rikio Matsumoto ${ }^{1, \dagger}$, Gavin R. Broad ${ }^{2, \ddagger}$ \\ I Osaka Museum of Natural History, Nagai Park 1-23, Higashisumiyoshi-ku, Osaka, 546-0034, JAPAN \\ 2 Natural History Museum, Cromwell Road, London SW7 5BD, UK \\ † urn:lsid:zoobank.org:author:9D30E868-4C4B-4F40-A6C8-781F185FA0E4 \\ † urn:lsid:zoobank.org:author:D06689DE-526F-4CFA-8BEB-9FB38850754A \\ Corresponding author: Rikio Matsumoto (rikio@mus-nh.city.osaka.jp)
}

Academic editor: Stefan Schmidt| Received 26 October 2009 | Accepted 23 November 2010 | Published 8 February 2011

urn:lsid:zoobank.org:pub:126C05BD-5E28-4942-BEF0-07FD4A5D5665

Citation: Matsumoto R, Broad G (2011) Discovery of Rodrigama Gauld in the Old World, with description of two new species (Hymenoptera, Ichneumonidae, Poemeniinae). Journal of Hymenoptera Research 20: 65-75. doi: 10.3897/ jhr.29.872

\begin{abstract}
Two new species of the genus Rodrigama Gauld are described: R. gauldi sp. n. from Ryukyus, Japan and $R$. takakuwai sp. n. from Taiwan. The genus Rodrigama was known from a single Costa Rican species and these new species are the first representatives of the genus in the Old World. The distribution of these species in the Palaearctic/Oriental Region reinforces the hypothesis that the Poemeniinae originated in the north temperate region and diversified into tropical regions, and that the Costa Rican $R$. gamezi is a relict of a group that once had a wider distribution. A key to the species of the genus is provided.
\end{abstract}

\section{Keywords}

Taxonomy, biogeography, distribution, Taiwan, Japan

\section{Introduction}

Wahl and Gauld (1998), in their phylogenetic analysis of Pimpliformes, demonstrated that Rodrigama occupies a basal position in the Poemeniinae and is the sister group to higher poemeniines, and accordingly bestowed tribal status (Rodrigamini) on the one 
species known at the time, Rodrigama gamezi Gauld. They regarded the genus Pseudorhyssa Merrill as the sister group to all other Poemeniinae and erected the tribe Pseudorhyssini. Pseudorhyssa is somewhat aberrant among the subfamily and Quicke et al. (2009) noted that it might deserve its own subfamily status. The apparently primitive genus, Rodrigama, was known from only a single species, $R$. gamezi, described from Costa Rica. The isolated distribution of this archaic genus in Mesoamerica was anomalous and did not fit the hypothesis of an Holarctic origin of the Poemeniinae (Wahl and Gauld 1998). Recently, two new species of Rodrigama have been found, one from the Ryukyu Archipelago, at the Oriental and Palaearctic boundary in Japan, and another from Taiwan.

\section{Materials and methods}

Type specimens of the two new species are preserved in the collection of Osaka Museum of Natural History (OMNH), except the holotype of $R$. takakuwai in the Taiwan Agriculture Research Institute, Taichung, Taiwan (TARI) and one paratype of $R$. gauldi in the Natural History Museum, London (BMNH). Three paratype specimens (two females and one male) of the genotype, Rodrigama gamezi Gauld, 1991, preserved in $\mathrm{BMNH}$, were examined for comparison. A scanning electron microscope (KEYENCE VE-7800) was used for detailed observation of external morphology of adults. The image of the specimen of $R$. gamezi was taken using a Canon EOS 450D digital camera and Pentax $50 \mathrm{~mm}$ macro lens. Several partially focused images were combined using Helicon Focus v. 4.80. Terminology of adult morphology follows Gauld (1991).

\section{Taxonomy}

\section{Genus Rodrigama Gauld}

Figs 1-20

Rodrigama Gauld 1991: 536 Type species: Rodrigama gamezi Gauld, by original designation.

Diagnosis. Rodrigama can be distinguished from all other poemeniines by either of the following: 1) paired antero-lateral and postero-lateral oblique grooves delimiting a central rhombic area on the second metasomal tergite; 2) fused tergite and sternite of the first metasomal segment and can be readily identified using Wahl \& Gauld's (1998) key to genera.

Description. Medium to large ichneumonids with fore wing length $12-26 \mathrm{~mm}$. Apical segment of flagellum tapered apically, with distal end narrowly truncate; eyes with inner margins parallel; lower face transverse, about $1.3 \times$ as broad as high at narrowest point; clypeus (Fig. 6) moderately large, not divided into basal and apical parts; clypeal margin thin, more or less indented, about $2 \times$ as wide as high; mandible (Fig. 
6) stout, with lower tooth slightly twisted forwards, entire mandible evenly tapered, bidentate, with lower tooth distinctly longer and stouter than upper tooth; posterior ocellus separated from eye by 1.4-1.7 $\times$ its maximum diameter; occipital carina (Figs $4,5)$ centrally narrowly incomplete, mediodorsally slightly dipped; upper part of gena smooth; head in dorsal view with genae of moderate length, barely narrowed behind eyes. Pronotum (Fig. 7) with a mediodorsal posteriorly directed tooth, with strongly impressed and transversely striate groove extending from upper hind corner down to lower corner; upper part of epomia weak and short. Mesoscutum highly polished, sparsely covered with fine punctures and pubescence, without transverse rugae; epicnemial carina present, with upper end reaching level of lower corner of pronotum. Propodeum (Figs 8, 18) quite short, convex, with longitudinal carinae weak or absent; pleural carina clearly discernible; propodeal spiracle oval. Metapleuron strongly convex; submetapleural carina complete. Fore wing (Fig. 16) with vein 3rs-m present, defining a shortly petiolate, very wide, irregularly rhombic areolet; vein Rs+M opposite or a little basad of cu-a; 2rs-m about as long as $\mathrm{M}$ between $2 \mathrm{rs}-\mathrm{m}$ and $2 \mathrm{~m}$-cu; $2 \mathrm{~m}$-cu with two bullae; Cu1b shorter than abscissa of $\mathrm{Cu} 1$ between $1 \mathrm{~m}$-cu and Cula; hind wing with 4-5 hamuli on costal margin proximally. Tarsal claws of both sexes simple, large, symmetrical, abruptly curved, with neither inner tooth nor spatulate bristles; hind leg very slender with tibia and tarsus particularly long and slender, with trochantellus not exceptionally long. Metasoma of female (Figs 1-3) moderately slender, fusiform; tergite 1 (Figs 11-12,19) very long and slender, dorsally smooth and polished, without lateromedian and lateral longitudinal carinae, with spiracles on promontories at centre of tergite; sternite 1 fused to tergite, anteriorly forming cylinder; glymma absent; tergites $2-4$ with grooves delimiting more or less rhombic central areas (Figs 1-3, 13, 20); last visible tergite extending into flat, shelf-like posterior prolongation which overhangs cerci (Fig. 14); sternites $2+$ to subgenital plate medially indented, latter with small median incision posteriorly; ovipositor moderately stout, projecting beyond subgenital plate by about $4.5 \times$ length of hind tibia, apex somewhat depressed, evenly tapered to point; lower valve dorsolaterally expanded to partially enclose upper, with distinct teeth (Fig. 15); male with metasoma slightly compressed; sternites evenly sclerotized, folded on mid-line; subgenital plate transverse, with posterior margin slightly concave.

Biological notes. Host unknown. Females of $R$. gamezi were observed flying around an old, dead tree, and one was collected whilst probing in an old beetle boring with its ovipositor (Gauld 1991).

\section{Key to species of Rodrigama}

1 Body black to reddish brown (Figs 1,2). Pronotum, mesoscutum and mesopleuron finely punctate (Figs 7, 17). Epicnemial carina short, absent on mesoscutum. Posterior transverse carina of propodeum absent or weakly in-

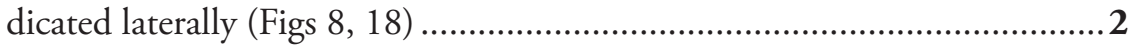

- Body yellowish/tan brown (Fig. 3). Pronotum, mesoscutum and mesopleuron almost entirely impunctate. Epicnemial carina present on mesoscutum. 
Posterior transverse carina of propodeum complete and strongly raised.

gamezi Gauld

2 Subalar prominence yellow (Fig. 1). First to fifth metasomal tergites brown to dark brown, with posterior whitish band (this band sometimes interrupted medially) (Fig. 1). Pronotum with weak, medially incomplete oblique groove from epomia to ventroposterior corner (Fig. 7). Area dorsal to this groove weakly punctate. First metasomal tergite gradually broadened posteriorly in dorsal aspect, with posterior end much broader than width at anterior 0.2 (Fig. 12)

gauldisp. n.

- Subalar prominence reddish brown, concolourous with rest of mesopleuron (Fig. 2). First to fifth metasomal tergites brown to reddish brown all over, never with posterior whitish band (Fig. 2). Pronotum with strongly impressed and transversely striate groove from epomia to ventroposterior corner (Fig. 17). Area dorsal to this groove rather strongly and densely punctate, slightly rugose. First metasomal tergite broadened at anterior 0.1 and slightly narrowed a little posterior to spiracle, with posterior end slightly broader than width at anterior 0.2 (Fig. 19) takakuwai sp. n.

\section{Rodrigama gamezi Gauld, 1991}

Fig. 3

Notes. Gauld's (1991) description is sufficient for recognition of $R$. gamezi; we add the following characters for comparison with the two species newly described here: epicnemial carina present on mesoscutum; mesopleural suture mostly smooth, with some faint transverse striations; posterior transverse carina of propodeum strongly raised; first metasomal sternite extending back to about half way between spiracle and posterior margin of tergite 1. A paratype $q$ is illustrated in Fig. 3.

\section{Rodrigama gauldi Matsumoto and Broad, sp. $\mathrm{n}$.}

urn:Isid:zoobank.org:act:D66BDEC2-E88C-4DCA-9F66-47E2884F3331

Figs 1, 4-16

Holotype + (Fig. 1), 21-27. iv.2008 (Malaise trap), Aha, (26²' $52^{\prime \prime N}, 128^{\circ} 16^{\prime} 09^{\prime \prime E}$ (WGS 84), 150m a.s.l.), Kunigami-son, Okinawa-jima, Okinawa Pref., Ryukyus, Japan (K. Sugino) [OMNH, TI-480].

Paratypes. 3ㅇ, 7-21.iv, 21-27.iv, 20-26.v, (OMNH), 1q, 14-20.v (BMNH), otherwise same data as holotype, collected in a Malaise trap operated by K. Sugino.

Diagnosis. This species can be distinguished from all congeners by the following: pronotum with weak, medially incomplete oblique groove from epomia to ventroposterior corner; area dorsal to this groove weakly punctate; subalar prominence yellow; first metasomal tergite with apical whitish band; tergites $2-5$ each 

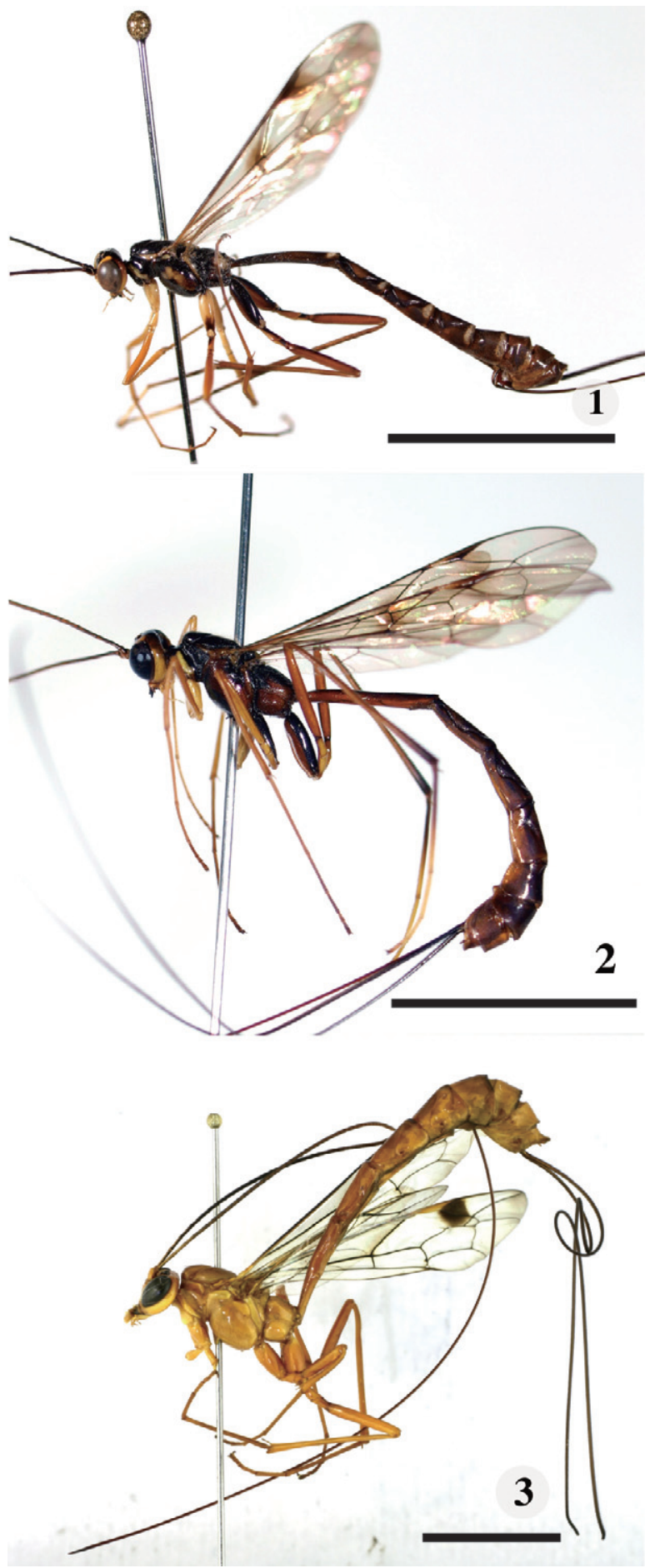

Figures I-3. Rodrigama spp., whole insect, lateral aspect. I R. gauldi sp. n. (holotype) $\mathbf{2}$ R. takakuwai sp. n. (holotype) 3 R. gamezi Gauld (paratype) (scale: $10 \mathrm{~mm}$ ). 
with paired whitish transverse markings apically, these markings sometimes fused dorso-medially.

Description. Female. Length of fore wing 12-13 mm. Malar space (Fig. 6) about $0.2 \times$ basal width of mandible. Pronotum (Fig. 7) with weak medially incomplete oblique groove from epomia to ventroposterior corner; area dorsal to this groove weakly punctate. Mesoscutum in front of scuto-scutellar groove $1.3 \times$ as long as wide in dorsal view; length of pubescence in centre of middle lobe about $1.5 \times$ as long as distance between adjoining punctures; mesopleuron moderately punctate anteriorly, rather finely and sparsely punctate posteriorly, rather densely covered with pubescence; mesopleural suture strongly transversely striate; propodeum (Fig. 8) moderately punctate, transversely striate dorsomedially, with anterior part of lateromedian longitudinal carina present (sometimes very short), without swelling above spiracle; posterior transverse carinae absent. First metasomal tergite (Fig. 12) gradually broadened posteriorly in dorsal aspect, $6.5 \times$ as long as posteriorly broad; posterior end of first metasomal sternite at posterior 0.35 between spiracle and posterior end of first metasomal tergite (Fig. 11); tergites 2-4 (Fig. 13) sparsely covered with fine punctures and minute pubescence.

Colouration (Fig. 1). Head largely yellow with following parts black: apical half of mandible, occiput, frons except narrow yellow stripe extending along inner orbit to top of eye, narrow stripe behind eye extending downwards and fading around middle of eye; labrum dark brown; gena brownish, darkened dorsally. Mesosoma black with following parts yellow: latero-ventral and postero-dorsal margins of pronotum, pair of longitudinal spots on mesoscutum, tegula, subalar prominence, central spot on mesopleuron, scutellum, postscutellum, propodeum posteriorly except black semicircular mark along posterior margin above insertion of metasoma. Legs yellowish brown. Coxa, trochanter and trochantellus of fore leg slightly paler. Middle coxa narrowly brownish apically above. Trochanter of middle leg longitudinally darkened on dorsal face. Hind coxa fulvous, dorsally black. Trochanter, trochantellus, femur of hind leg darkened dorsally. Second to fourth tarsomeres slightly paler. Wings hyaline, with distinct brownish spots around junction of $\mathrm{cu}-\mathrm{a}$ and $\mathrm{Cu} 1$, and on Rs+2r below pterostigma. Metasomal tergites brown, darkened on first tergite basally, tergites $2-4$ along oblique grooves; first metasomal tergite with apical whitish band, tergites 2-5 each with paired whitish transverse markings apically (Fig. 1), these markings sometimes fused dorso-medially. Ovipositor brown, sheath black.

Male. Unknown.

Distribution. Japan: Ryukyus (Okinawa-jima).

Etymology. We are very pleased to be able to name this species after the late Dr Ian Gauld, who recognised Rodrigama as a basal poemeniine and who has left such a rich legacy of ichneumonid taxonomy. It is a great pity that he did not live long enough to see these additional Rodrigama species.

Biological notes. Host unknown. Females were caught in Malaise traps positioned alongside paths in mature evergreen and broad-leaved forest of low altitude. Adult wasps were captured in April, May and June, suggesting spring emergence and early summer activity of adult wasps. 


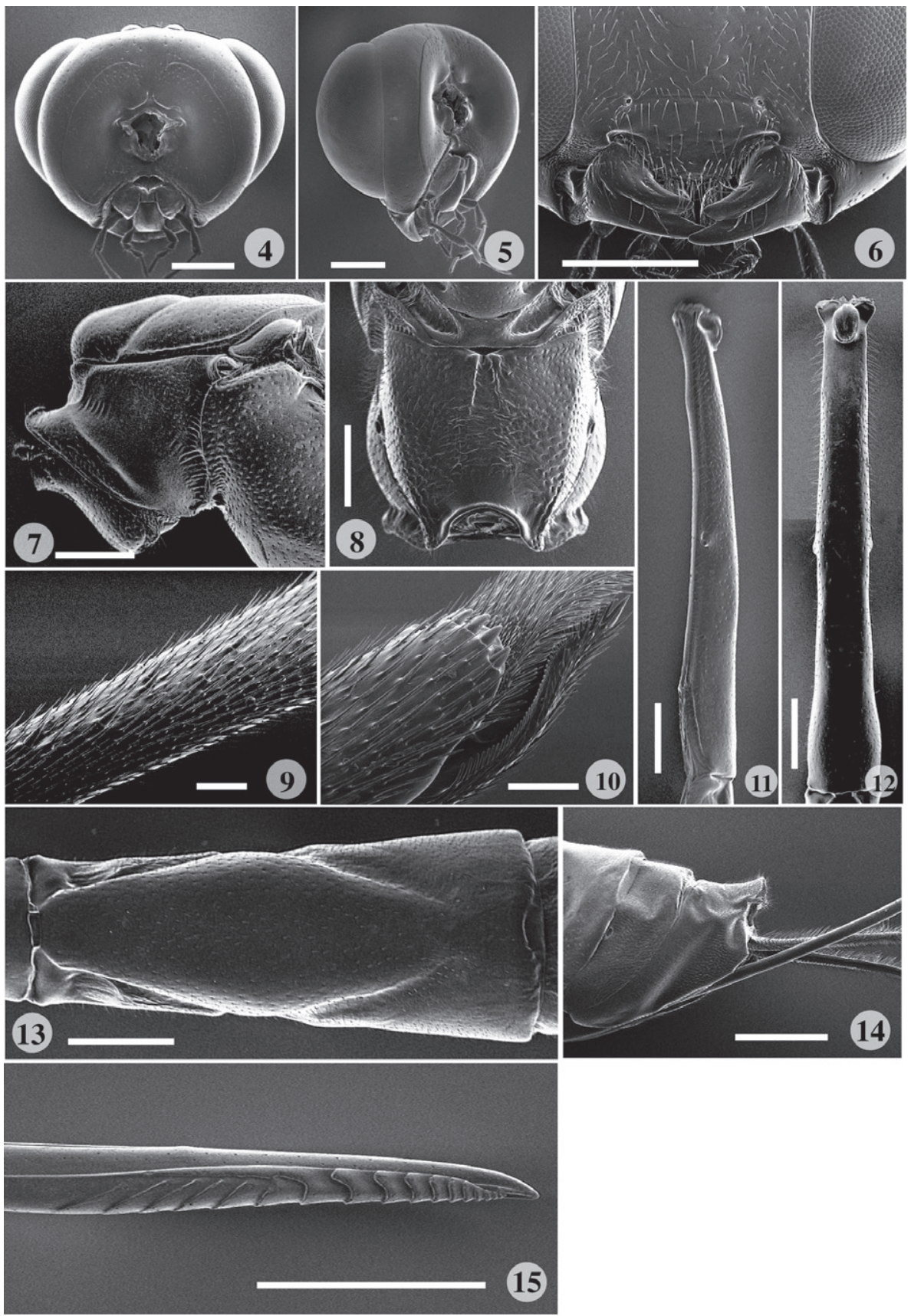

Figures 4-I5. Scanning electron micrographs of $R$. gauldi sp. n. (paratype). 4 Head, posterior aspect $\mathbf{5}$ head, postero-lateral aspect $\mathbf{6}$ clypeus, malar space and mandible, anterior aspect $\mathbf{7}$ anterior part of mesosoma, lateral aspect $\mathbf{8}$ propodeum, dorsal aspect; $\mathbf{9 - 1} \mathbf{0}$ fore tibia $\mathbf{9}$ dorsal aspect $\mathbf{I} \mathbf{0}$ stout spines distal end; I I-I $\mathbf{2}$ first metasomal tergite I I lateral aspect $\mathbf{I} \mathbf{2}$ dorsal aspect $\mathbf{I} \mathbf{3}$ second metasomal tergite, dorsal aspect $\mathbf{I} \mathbf{4}$ posterior end of metasoma, lateral aspect $\mathbf{I} \mathbf{5}$ ovipositor tip, lateral aspect (scale: $0.1 \mathrm{~mm}$ for $\mathbf{9}$, $10 ; 0.5 \mathrm{~mm}$ for $4-8,13,15 ; 1.0 \mathrm{~mm}$ for $11,12,14)$. 


\section{Rodrigama takakuwai Matsumoto and Broad, sp. n.}

urn:lsid:zoobank.org:act:2077A395-728C-473B-9E9C-39A2BA43DAB2

Figs 2, 17-20

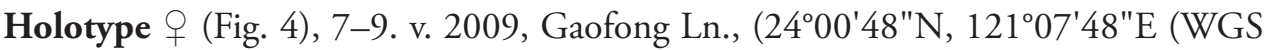
84), 1,400m a.s.l.), Ren-ai, Ln., Ren-ai t., Nantou, Taiwan, (Masatoshi Takakuwa) [TARI, 19-8].

Paratypes. [Taiwan] 3ð, 9. iv. 1996, Kuantaoshan, Nantou (R.M.) (OMNH).

Diagnosis. This species can be distinghished from all congeners by the following: Pronotum with strongly impressed and transversely striate groove from epomia to ventroposterior corner; area dorsal to this groove rather strongly and densely punctate; subalar prominence reddish brown, without yellow mark; metasomal tergites entirely brown to reddish brown, without whitish transverse markings apically.

Description. Female. Length of fore wing $12 \mathrm{~mm}$. Malar space about $0.2 \times$ basal width of mandible. Pronotum (Fig. 17) with strongly impressed and transversely striate groove from epomia to ventroposterior corner; area dorsal to this groove rather strongly and densely punctate, slightly rugose. Mesoscutum in front of scuto-scutellar groove $1.5 \times$ as long as wide in dorsal view; length of pubescence at centre of middle lobe about $3 \times$ as long as distance between adjoining punctures; mesopleuron moderately punctate anteriorly, rather finely and sparsely punctate posteriorly, rather densely covered with pubescence; mesopleural suture strongly transversely striate; propodeum (Fig. 18) moderately punctate, transversely striate dorsomedially, with anterior part of lateromedian longitudinal carina present (sometimes very short), without swelling above spiracle; posterior transverse carinae very weak and incomplete. First metasomal tergite (Fig. 19) broadened at anterior 0.1 , slightly narrowed a little posterior to spiracle, with posterior end slightly broader than width at anterior 0.2 ; posterior end of first metasomal sternite at posterior 0.35 between spiracle and posterior end of first metasomal tergite; tergites $2-4$ sparsely covered with fine punctures and minute pubescence.

Colouration (Fig. 2). Head largely yellow with following parts black: apical half of mandible, frons except narrow yellow stripe extending along inner orbit to top of eye, dorso-median area of vertex and occiput; gena brown above. Pronotum black with yellow elongate marking next to antero-lateral margin on both side, with upper margins yellow posteriorly. Mesoscutum black with pair of yellow longitudinal spots; mesopleuron reddish brown, area between subalar prominence and speculum black; mesosternum black; scutellum black with median yellow triangular marking; scutellum yellow; propodeum dark brown, ventrally reddish brown below lateral longitudinal carina behind spiracle; metapleuron reddish brown with anterior margin blackish. Legs yellowish brown. Coxa, trochanter and trochantellus of fore leg slightly paler. Middle coxa black dorsally. Hind coxa fulvous, dorsally black. Trochanter darkened dorsally, hind femur and tibia reddish brown, latter darkened apically; basal 0.6 of first tarsomere brown. Wings hyaline, slightly tinged with brown; pterostigma brown, with faint brownish spots around junction of of $\mathrm{cu}-\mathrm{a}$ and $\mathrm{Cu} 1$, and on $\mathrm{Rs}+2 \mathrm{r}$ below pterostigma. Metasomal tergites brown to reddish brown. Ovipositor brown, sheath black. 

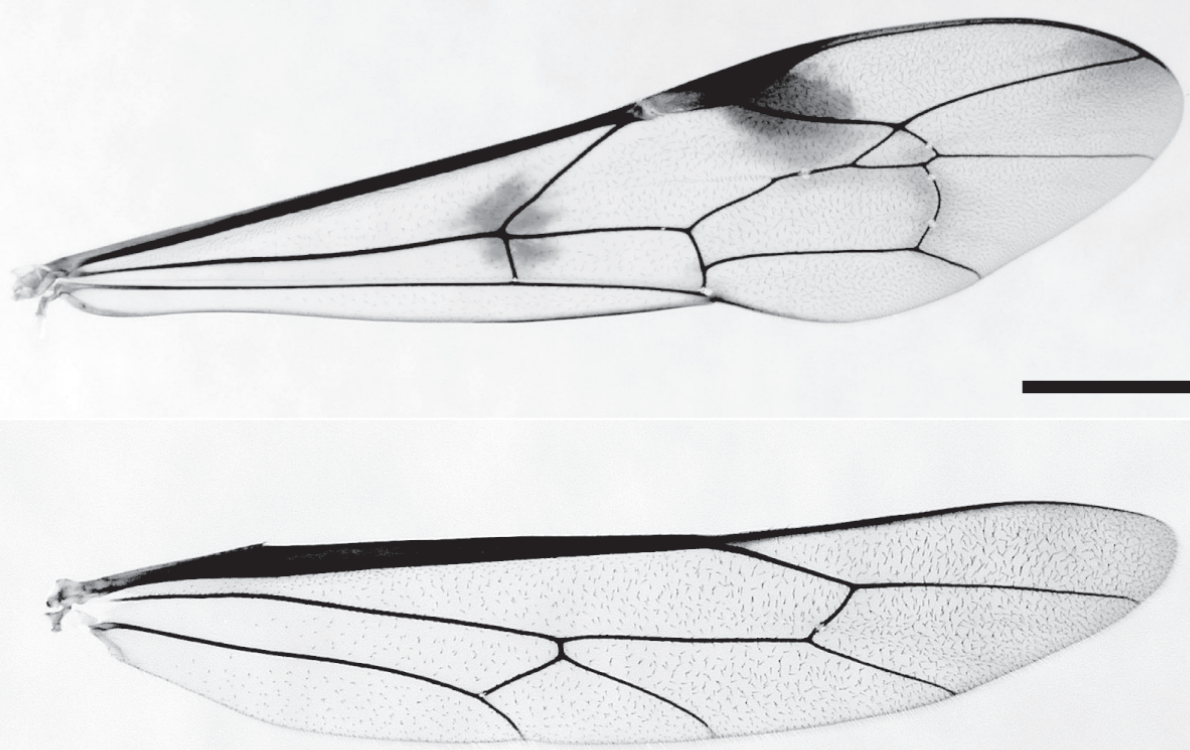

Figure 16. Fore and hind wing of $R$. gauldi sp. n. (paratype) (scale: $2 \mathrm{~mm}$ ).
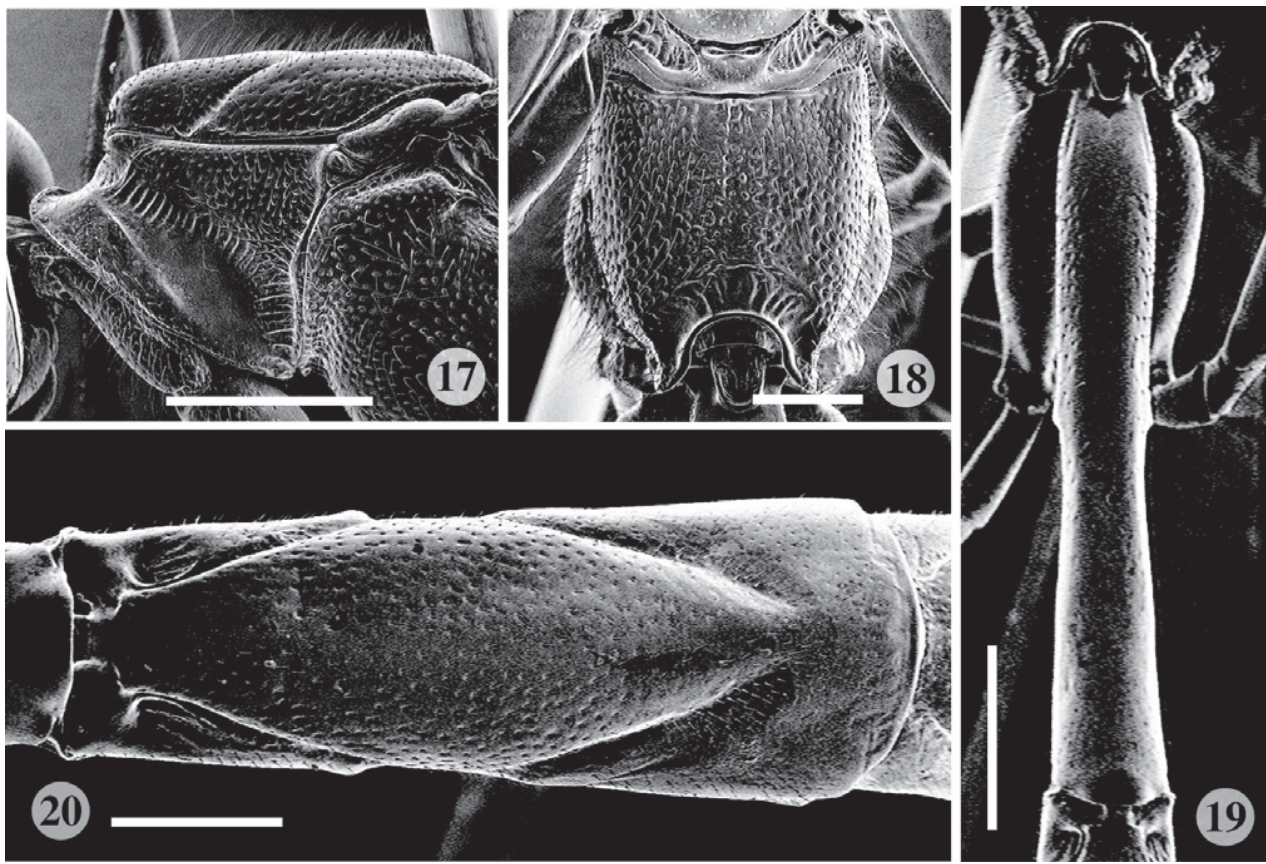

Figures 17-20. Scanning electron micrographs of $R$. takakuwai (holotype). 17 Anterior part of mesosoma, lateral aspect $\mathbf{1 8}$ propodeum, dorsal aspect $\mathbf{1 9}$ first metasomal tergite, dorsal aspect $\mathbf{2 0}$ second metasomal tergite, dorsal aspect. (scale: $0.5 \mathrm{~mm}$ for 18,$20 ; 1.0 \mathrm{~mm}$ for 17,19 ). 
Male. Similar to female but smaller. Length of fore wing $8.0-9.5 \mathrm{~mm}$. Body colour similar to that of female; dark stripe on dorsal face of mid coxa reduced; hind femur blackish dorsally; metasoma reddish brown; fore wing without brown spot.

Distribution. Taiwan (central mountainous region).

Etymology. This species is named after Dr Masatoshi Takakuwa who collected the Holotype.

Biological notes. Host unknown. Adult wasps were hand netted in mature forest of rather high altitude $(1,100-1,400 \mathrm{~m}$ a.s.l.). Both the preceding species and this Taiwanese species have only been found in mature forest.

\section{Discussion}

\section{Redefinition of the genus}

In addition to the characters listed in the original description of Rodrigama, the following characters are shared by all three known species of Rodrigama and also serve to define the genus: apical segment of flagellum tapered apically, with distal end narrowly truncate; vein $2 \mathrm{~m}$-cu of fore wing with two bullae; apex of ovipositor somewhat depressed. On the other hand, the following characters have now been found to be variable within the genus and cannot be used to define the genus: epicnemial carina present ventrally; propodeum with lateral and lateromedian carinae discernible from anterior edge to quite strongly raised posterior transverse carina; first metasomal sternite extending back to a level about half way between spiracle and posterior margin of tergite. These are characters unique to $R$. gamezi. Wahl and Gauld (1998) recognized the spiracles of the first metasomal tergite on promontories as an autapomorphy of the genus. This character is shared by all three members of the genus and reconfirmed here as an autapomorphy. Wahl and Gauld (1998) incorrectly coded the epicnemial carina as absent in $R$. gamezi and were incorrect also in their tribal diagnosis of Rodrigamini, as the epicmnemial carina is present in all three species of the genus. Incidentally, the occipital carina of $R$. gamezi was coded as complete but it is centrally narrowly incomplete.

\section{Distribution of the genus}

Wahl and Gauld (1998) hypothesized that the Pimpliformes arose in the north temperate region and diversified into the tropical regions, particularly into the Old World tropics, and latterly into the New World tropics. This scenario is well supported by the distributions of Acaenitinae and Rhyssinae (Wahl and Gauld 1998; Yu et al. 2005), in which the most derived genera are primarily tropical and the less derived are Holarctic. This pattern is also mostly upheld by the Poemeniinae, in which most of the more 
plesiomorphic genera, i.e. Pseudorhyssa, Poemenia, Deuteroxorides and Neoxorides, are Palaearctic or Holarctic, whereas the derived lineages, Guptella + Cnastis + Ganodes, Eugalta, have radiated into the more equatorial latitudes (Podoschistus is present in the temperate and Oriental realms). Wahl and Gauld (1998) noted an exception in that the Rodrigamini, the second basal-most branch of the Poemeniinae, was represented by one Neotropical species. They suggested that the Costa Rican species was a relict of a group that once had a wider distribution. Discovery of these additional species of Rodrigama in the Ryukyus and Taiwan, at the Oriental and Palaearctic boundary, strongly supports their hypothesis. It suggests a former, wider distribution of the genus in the Holarctic region, now surviving only on the Pacific rim of the Old World and in Central America. A similarly disjunct distribution is known, within Ichneumonidae, for the xoridine genus Aplomerus (Townes 1969; Watanabe and Matsumoto 2010; Broad in prep.), known from North America, Japan and Vietnam.

\section{Acknowledgements}

The first author would like to express his sincere gratitude to Kouichi Sugino (Kunigami Tourism Association, Okinawa) and Masatoshi Takakuwa (Kanagawa Prefectural Museum of Natural History) for offering valuable materials and information. Thanks are also due to Manabu Yashiro (Kakogawa City) for his help in sorting ichneumonids from numerous Malaise trap samples. Two reviewers provided constructive criticism that improved this paper.

\section{References}

Gauld ID (1991) Ichneumonidae of Costa Rica 1. Memoirs of the American Entomological Institute 47: 1-589.

Quicke DLJ, Laurenne NM, Fitton MG, Broad GR (2009) A thousand and one wasps: a $28 \mathrm{~S}$ rDNA and morphological phylogeny of the Ichneumonidae (Insecta: Hymenoptera) with an investigation into alignment parameter space and elision. Journal of Natural History 43: $1305-1421$.

Townes HK (1969) The genera of Ichneumonidae, part 1. Memoirs of the American Entomological Institute 11: 1-300.

Wahl DB, Gauld ID (1998) The cladistics and higher classification of the Pimpliformes (Hymenoptera: Ichneumonidae). Systematic Entomology 23: 265-298.

Watanabe K, Matsumoto R (2010). Disjunctive distribution of the basal genus Aplomerus (Hymenoptera: Ichneumonidae: Xoridinae) in East Asia and North America, with a new species from Japan. Entomological Science 13: 375-380.

Yu D, van Achterberg K, Horstmann K (2005) World Ichneumonoidea 2004. Taxonomy, biology, morphology and distribution. [CD/DVD computer program]. Taxapad ${ }^{\ominus}$, Vancouver, Canada. 